\title{
Niche similarities among introduced and native mountain ungulates
}

\author{
B. Lowrey, ${ }^{1,6}$ R. A. Garrott, ${ }^{1}$ D. E. McWhirter, ${ }^{2}$ P. J. White, ${ }^{3}$ N. J. DeCesare, ${ }^{4}$ and S. T. Stewart ${ }^{5}$ \\ ${ }^{1}$ Department of Ecology, Montana State University, Bozeman, Montana 59717 USA \\ ${ }^{2}$ Wyoming Game and Fish Department, Cody, Wyoming 82414 USA \\ ${ }^{3}$ Yellowstone Center for Resources, Yellowstone National Park, National Park Service, Mammoth, Wyoming 82190 USA \\ ${ }^{4}$ Montana Department of Fish, Wildlife, and Parks, Missoula, Montana 59804 USA \\ ${ }^{5}$ Montana Department of Fish, Wildlife, and Parks, Red Lodge, Montana 59068 USA
}

\begin{abstract}
The niche concept provides a strong foundation for theoretical and applied research among a broad range of disciplines. When two ecologically similar species are sympatric, theory predicts they will occupy distinct ecological niches to reduce competition. Capitalizing on the increasing availability of spatial data, we built from single species habitat suitability models to a multispecies evaluation of the niche partitioning hypothesis with sympatric mountain ungulates: native bighorn sheep (BHS; Ovis canadensis) and introduced mountain goats (MTG; Oreamnos americanus) in the northeast Greater Yellowstone Area. We characterized seasonal niches using two-stage resource selection functions with a used-available design and descriptive summaries of the niche attributes associated with used GPS locations. We evaluated seasonal similarity in niche space according to confidence interval overlap of model coefficients and similarity in geographic space by comparing model predicted values with Schoener's $D$ metric. Our sample contained 37,962 summer locations from 53 individuals $(\mathrm{BHS}=31, \mathrm{MTG}=22)$, and 79,984 winter locations from 57 individuals $(\mathrm{BHS}=35$, MTG $=22$ ). Slope was the most influential niche component for both species and seasons, and showed the strongest evidence of niche partitioning. Bighorn sheep occurred on steeper slopes than mountain goats in summer and mountain goats occurred on steeper slopes in winter. The pattern of differential selection among species was less prevalent for the remaining covariates, indicating similarity in niche space. Model predictions in geographic space showed broad seasonal similarity (summer $D=0.88$, winter $D=0.87$ ), as did niche characterizations from used GPS locations. The striking similarities in seasonal niches suggest that introduced mountain goats will continue to increase their spatial overlap with native bighorn. Our results suggest that reducing densities of mountain goats in hunted areas where they are sympatric with bighorn sheep and impeding their expansion may reduce the possibility of competition and disease transfer. Additional studies that specifically investigate partitioning at finer scales and along dietary or temporal niche axes will help to inform an adaptive management approach.
\end{abstract}

Key words: bighorn sheep; mountain goat; niche; Oreamnos americanus; Ovis canadensis; resource selection function; Yellowstone.

\section{INTRODUCTION}

First described by Joseph Grinnell (1917), the niche concept links environmental variables and conditions to the distribution and fitness of species, and continues to provide a strong foundation for theoretical and applied research among a broad range of disciplines (Chase and Leibold 2003). Hutchinson (1957) later formalized the ecological niche as a multi-dimensional hypervolume that completely defines a species' ecological properties required for positive growth, that is, the fundamental niche. While conceptually appealing, fundamental niches contain a virtually infinite number of axes and may be a purely theoretical construct (Panzacchi et al. 2014). The realized niche accounts for changes in a species' fundamental niche attributed to interspecific interactions and provides a more interpretable subset of the all-encompassing hypervolume (Hutchinson 1957). The striking increase in animal location data and environmental variables characterized in Geographic

Manuscript received 2 November 2017; revised 31 January 2018; accepted 21 February 2018. Corresponding Editor: Jacob R. Goheen.

${ }^{6}$ E-mail: blakelowrey@montana.edu
Information Systems (GIS) has strengthened ecologists' ability to estimate realized niches in both environmental and geographic space (Elith et al. 2006, Hirzel and Le Lay 2008, Soberón and Nakamura 2009). Largely reliant on abiotic niche components, habitat suitability models (e.g., resource selection functions [Manly et al. 2002]; ecological niche factor analyses [Hirzel et al. 2002]; and species distribution models [Phillips and Dudík 2008, among others]) are being increasingly used to describe the characteristics, interactions, and evolution of realized niches with relevance to theoretical and applied contexts, and represent a novel approach to examine the niche characteristics of sympatric species (Hirzel and Le Lay 2008).

The long and varied history of the niche concept has resulted in a multitude of definitions within the field of ecology (Chase and Leibold 2003). Most notably, the term "niche" has been confusingly used to address two distinct concepts, Grinnellian (i.e., habitat requirements of a species) and Eltonian (i.e., a species ecological role) niches (Hirzel and Le Lay 2008). Moreover, while Hutchinson's fundamental and realized niches provided a unifying and quantifiable framework, the measures added an additional interpretation to the niche concept (Chase and Leibold 
2003). Herein we defined niche according to Hutchinson (1957), and quantified realized niches largely reliant on Grinnellian (i.e., abiotic) habitat components (Hirzel and Le Lay 2008).

While the advances in GPS technology have resulted in dramatic increases in species-specific habitat suitability models, far fewer studies have used GPS data to quantify niche similarity among sympatric species. When two ecologically similar species are sympatric, the niche partitioning hypothesis predicts they will occupy distinct niches to reduce interference (direct) and exploitive (indirect) competition (Pianka 1981). For example, MacArthur's Warblers, five species of insectivorous wood warblers, were observed to alter foraging times and behavior in the presence of one another to exploit different food types and reduce interspecific competition and potential competitive exclusion (MacArthur 1958). Niche partitioning is realized through the behavioral process of resource selection, which results in spatiotemporal separation in environmental, and in turn, geographic space (Schoener 1974). As the use of GPS technology continues to increase across a broad range of taxa with overlapping distributions (Hebblewhite and Haydon 2010), ecologists have an opportunity to expand from single species habitat suitability models to applied and theoretical research among relevant taxonomic groups (Hirzel and Le Lay 2008). For example, habitat suitability models of native and introduced species can be used to characterize their niche dynamics and evaluate the possibility for competition when the two species are sympatric. Moreover, in systems with introduced species, there is an opportunity to study interspecific competition and the extent to which natural selection has partitioned niches of related taxa. Herein, we integrate tools from the habitat suitability and niche literatures and develop a novel framework to evaluate the niche partitioning hypothesis with native and introduce mountain ungulates in the Greater Yellowstone Area (GYA).

Mountain goats (Oreamnos americanus) are an iconic species with strong associations with steep and rugged terrain (Chadwick 2002). Beginning in the mid-1900s, state wildlife agencies used translocation programs to expand the distribution of mountain goats and increase hunting opportunities throughout western North America (Côté and Festa-Bianchet 2003). These efforts have largely been successful and greatly expanded the distribution of mountain goats both within and beyond their native ranges (Côté and Festa-Bianchet 2003, Flesch et al. 2016). Although the expanding mountain goat population in nonnative areas has been embraced by some natural resource agencies, there is concern that encroachment into areas occupied by native bighorn sheep (Ovis canadensis) may be detrimental to regional restoration efforts (Adams et al. 1982, Lowrey et al. 2017). Bighorn sheep have struggled to rebound to historic numbers since the early 1900 s and much of their historic range remains unoccupied (Buechner 1960). Moreover, bighorn sheep are negatively impacted by respiratory pathogens and habitat loss, particularly on low-elevation winter ranges, and largely occur within restored populations numbering fewer than 100 individuals (Buechner 1960, Cassirer et al. 2017). Because of the general propensity of both species to inhabit rugged, mountainous terrain, there is potential for increased spatial overlap as mountain goats continue to expand their nonnative range (Lowrey et al. 2017) and the possibility that competition (Reed 2001) and disease transfer (Gross 2001) will be detrimental to native bighorn sheep.

Because bighorn sheep and mountain goats have long occurred on sympatric native ranges within portions of western North America one would expect to observe niche partitioning as a likely consequence of their history on sympatric ranges (MacArthur 1958, Pianka 1981), although this prediction has not been directly evaluated. A conceptual model generalized across allopatric study areas suggested that mountain goats occur within the extreme end of some niche components (i.e., terrain steepness, ruggedness, snow cover, etc.) when compared to bighorn sheep (Adams et al. 1982). As mountain goats continue to expand their nonnative ranges, there is a need to understand their niche dynamics and evaluate the possibility for introduced mountain goats to alter behavioral processes of native bighorn sheep.

The GYA represents one of the largest relatively intact temperate ecosystems in the world (Keiter and Boyce 1994), and provides a rare opportunity to describe the niche characteristics of native bighorn sheep and sympatric introduced mountain goats (Lowrey et al. 2017). From an initial introduction of 170 animals to nine sites over 28 yr (1942-1970), mountain goats have expanded their distribution and grown to a minimum population estimate of 1,648 individuals within the GYA (Flesch et al. 2016). The northeast portion of the GYA, where introductions were first initiated and most concentrated, is nearly completely colonized by mountain goats with minimum counts of 632 individuals (Flesch et al. 2016). Herds of native bighorn sheep occur within and adjacent to the expanding mountain goat population throughout the GYA. Although the demographic performance of bighorn sheep within the GYA is varied, the northeastern portion represents an intact and robust population that was never extirpated, has no documented widespread disease related die-offs, and continues to show relatively stable demographic performance (Buechner 1960, Butler et al. 2017).

We tested the niche partitioning hypothesis through characterizing the realized niches of native bighorn sheep and introduced mountain goats within sympatric ranges of the GYA, and in so doing, built from single species habitat suitability models to interspecific research that broadens the ecological understanding of both species within an applied context. We hypothesized there would be broad similarity between summer niches as both species generally transition from relatively low-elevation winter ranges to high-elevation mountain environments following regional phenological patterns (Varley 1994, DeCesare and Pletscher 2006, Lowrey et al. 2017). In winter, we hypothesized that differing strategies among species would result in dissimilar niches. Although recent work suggests that wintering strategies can be varied with some individuals overwintering at high elevations (Courtemanch et al. 2017), bighorn sheep tend to occupy broad, low-elevation winter ranges (Festa-Bianchet 1988). Mountain goats, in contrast, select for steep cliffs at intermediate elevations, which more consistently shed snow 
and are patchily distributed on the landscape (Chadwick 2002, Lowrey et al. 2017).

\section{Methods \\ Study area}

The study area was located within the northeast GYA of southwest Montana and northwest Wyoming, and was characterized by rugged, mountainous topography with elevations ranging from 1,200 to $3,800 \mathrm{~m}$ above sea level and average annual precipitation of $130 \mathrm{~cm}$. The region experiences harsh, cold winters with snow persisting into the summer months at higher elevations. Land ownership is dominated by federally managed lands within designated wilderness areas, apart from valley bottoms, many of which were privately owned. The region has a strong predator population and hosts all native large carnivore species, including grizzly (Ursus arctos horribilis) and black bears (Ursus americanus), wolves (Canis lupus), coyotes (Canis latrans), mountain lions (Puma concolor), bobcat (Lynx rufus), wolverines (Gulo gulo), and Golden Eagles (Aquila chrysaetos). While there are only scant records of regional predation on mountain goats, bighorn sheep comprise a relatively small proportion of mountain lion and wolf diets regionally (Stahler et al. 2006, Elbroch et al. 2013). Included in the study area were sympatric bighorn sheep and mountain goat populations (Fig. 1). Ninety-six mountain goats were introduced within and adjacent to the study area from the early 1940 s to the mid-1950s. Mountain goats have continued to increase in abundance and distribution since their initial introductions (Laundré 1990, Flesch et al. 2016) and have been sympatric with bighorn sheep for approximately $50 \mathrm{yr}$ (Lemke 2004). Bighorn sheep and mountain goat minimum counts were 1,500 and 632 individuals in 2016, respectively (Flesch et al. 2016, McWhirter 2016, MFWP 2016). Within the study area, bighorn sheep were never extirpated or augmented, and have maintained relatively stable population demographics with modest harvest rates and nearly ubiquitous presence of respiratory pathogens in sampled animals (Butler et al. 2017).

\section{Data collection and censoring}

From 2012 to 2016, we used ground darting and helicopter net gunning to capture bighorn sheep and mountain goats, primarily during winter. We targeted adult females of both species though some mature male mountain goats were also included. All captured animals were fitted with both a store-on-board GPS (Telonics TGW-4400-2 or TGW-44003; Telonics, Mesa, AZ, USA) and VHF (Telonics MOD401-1) radio collar, enabling the acquisition of fine-scale spatiotemporal data as well as additional survival monitoring once the GPS collar released from the animal (Lowrey et al. 2017). The collars collected GPS locations at 4-, 5-, or 6-h intervals. All animals were captured and handled according to protocols approved by the Montana State University Institutional Animal Care and Use Committee (permits 2011-17, 2014-32).

We defined summer (3 July to 4 October) and winter (18 November to 15 May) periods using migration parameters

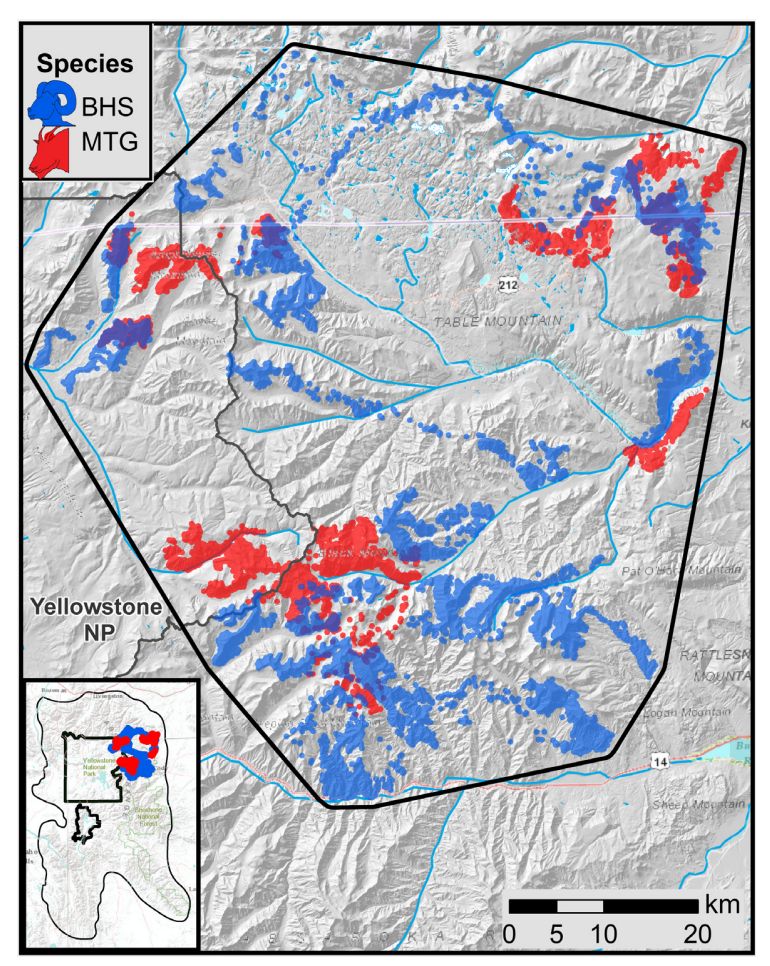

FIG. 1. GPS locations of 34 bighorn sheep (BHS) and 23 mountain goats (MTG) instrumented in the northeastern Greater Yellowstone Area (GYA), USA, 2012-2017. The study area (black polygon) was defined with a buffered minimum convex polygon around all locations.

estimated from nonlinear regression modeling of netsquared displacement (Bunnefeld et al. 2011, Spitz et al. 2017) and censored location data collected during the population-mean migratory periods to minimize the additional "noise" associated with transitional movements between summer and winter ranges (Appendix S1). We censored imprecise locations from the data set by removing GPS locations with a horizontal dilution of precision (HDOP) $>10$ (D'eon and Delparte 2005) and/or an estimate of horizontal error (included in Generation 4 Telonics data files) $>60 \mathrm{~m}$ (Lowrey et al. 2017). After censoring, the mean species-season fix success rates were $\geq 87 \%$ (Appendix S2).

\section{Realized niche components}

We used covariates (Table 1) supported by previous habitat studies of allopatric populations to characterize the realized niches of bighorn sheep and mountain goats. Terrain attributes included elevation (Ele), slope, slope variance (SlopeVar) calculated as the variance of slope values within the eight-cell neighborhood surrounding each grid cell of a $10 \mathrm{~m}$ DEM (USGS 2009, DeVoe et al. 2015), and three measures of distance to steep terrain (DST, akin to distance to escape terrain; DeCesare and Pletscher 2006), defined as slopes $\geq 30^{\circ}, 40^{\circ}$, and $50^{\circ}$. Indices of vegetation and forage abundance included canopy cover (CanCov; Homer et al. 2015) and time integrated normalized difference vegetation index (NDVI; USGS EROS Center 2016), respectively. To characterize heat load, we transformed aspect into a 
biologically interpretable covariate by taking the inverse cosine of the angle $-35^{\circ}$ (AspectCos; Cushman and Wallin 2002). This transformation changed the axis from northsouth to north-northeast-south-southwest and ranged from -1 to 1 , respectively. Lastly, snow water equivalent (SWE; NOHRSC 2004) indexed winter snow.

We included multiple spatial grains and functional forms for selected covariates to allow for differences in interspecific behavioral processes characteristic of niche partitioning and generated hypotheses regarding the strength, direction, and form of each covariate based on previous studies (Table 1). In addition, we incorporated the "space of influence" on animal decisions regarding resource selection (i.e., the multigrain resource selection function; Laforge et al. 2015). The multi-grain approach formalizes the concept that an animal's choice to select a given spatial location may not result solely from the attributes in the immediate vicinity (e.g., minimum resolution of the data), but may also be influenced by a broader region (e.g., the "space of influence"; Laforge et al. 2015). In addition to the minimal resolution of the data (i.e., 30 and $250 \mathrm{~m}$; Table 1), we performed neighborhood analyses within 500-, and 1,000-m circular buffers to evaluate alternative areas of influence and their relevance to mountain ungulate niches. We restricted our analysis of multiple grains to covariates that could be visually perceived to capture the behavioral processes driving resource selection. Covariates that are not directly perceived by animals (e.g., elevation), and therefore do not elicit a response, were not evaluated at multiple grains (Table 1; Lowrey et al. 2017).

\section{Seasonal niche characterizations}

We employed a variety of techniques to characterize the seasonal realized niches of sympatric mountain ungulates in niche and geographic space. First, we characterized seasonal niches employing a used-available design (i.e., Design II; Manly et al. 2002) where individual GPS locations represented the "used" set and "availability" was sampled within a shared study area-level minimum convex polygon (MCP; Fig. 1) buffered by the 95\% step length between consecutively acquired locations (Laforge et al. 2015). We used the same availability extent for each species and season, and employed a two-stage approach to move from individualbased models to a single mean population resource selection function (RSF) for each season, which approximated the seasonal realized niches (Marzluff et al. 2004, Fieberg et al. 2010). We treated individual as the experimental unit (White and Garrott 1990) and fit separate models for each individual-season combination with separate random samples of available points at a 1:10 (used:available) ratio (Appendix S3).

Beginning with individual univariate models, we used a tiered approach to identify the most explanatory functional form and/or spatial grain for covariates where multiple forms and/or grains were evaluated (Table 1). For each individual, we identified the top-ranked forms and grains using $\mathrm{AIC}_{\mathrm{c}}$ (Burnham and Anderson 2002). To generate a single model structure at the population-level for each species-season, we selected the form and grain for each covariate with the highest number of top-ranked occurrences when summed within the species-season groupings. We retained all covariates from the individual models, but allowed the form, grain size, and DST definition $\left(30^{\circ}, 40^{\circ}\right.$, or $50^{\circ}$ slopes $)$ to vary between species and seasons (Marzluff et al. 2004, Sawyer et al. 2009). When there was a tie in the top-ranked grain size, we subjectively defaulted to the smaller unit. We then combined covariates from the univariate models with those not evaluated with multiple forms and/or grains to evaluate collinearly. We maintained a Pearson's correlation coefficient of $r<|0.6|$ by selecting the least collinear form and/or grain size rather than using the top-ranked form and/ or grain size from the univariate results. In addition, we removed covariates if the correlation coefficient could not be reduced below the $|0.6|$ threshold for any covariate

TABle 1. Covariates used to approximate the realized niches of sympatric bighorn sheep (BHS) and mountain goats (MTG), GYA, 2012-2017.

\begin{tabular}{|c|c|c|c|c|c|}
\hline \multirow[b]{2}{*}{ Abbreviation } & \multirow[b]{2}{*}{ Description } & \multirow[b]{2}{*}{ Form } & \multirow[b]{2}{*}{ Spatial grain $\dagger$} & \multicolumn{2}{|c|}{ Hypothesized relationship (summer, winter) } \\
\hline & & & & MTG & BHS \\
\hline ELEV & elevation $(\mathrm{m})$ & $\mathrm{Li}$ & 30 & pos, neg & pos, neg \\
\hline SLP & slope (degrees) & $\mathrm{Li}, \mathrm{Sq}$ & $30,500,1,000$ & pos, pos & pos, neg \\
\hline SlopeVart & slope variance: standard deviation ${ }^{2}$ of SLP & Li, Ps & $30,500,1,000$ & pos, pos & pos, neg \\
\hline DST & $\begin{array}{l}\text { distance to steep terrain: Euclidian distance } \\
\text { from slopes } \geq 30^{\circ}, 40^{\circ}, 50^{\circ}\end{array}$ & $\mathrm{Li}$ & $30,500,1,000$ & neg, neg & neg, neg \\
\hline CanCov & canopy cover $(\%)$ & $\mathrm{Li}$ & $30,500,1,000$ & neg, neg & neg, neg \\
\hline NDVI & $\begin{array}{l}\text { time integrated NDVI: mean daily (interpolated) } \\
\text { integration of NDVI above the baseline for the } \\
\text { duration of the growing season from } 2011 \text { to } 2014\end{array}$ & $\mathrm{Li}$ & $250,500,1,000$ & pos, pos & pos, pos \\
\hline AspectCos & the inverse cosine of aspect minus $35^{\circ}$ & $\mathrm{Li}$ & 30 & pos, neg & pos, neg \\
\hline SWE & $\begin{array}{l}\text { snow water equivalent: mean Dec-Jan from } \\
2011 \text { to } 2014\end{array}$ & $\mathrm{Li}$ & 1,000 & na, neg & na, neg \\
\hline
\end{tabular}

Notes: Boldface type for the hypothesized relationship (positive [pos], negative [neg], or not applicable [na]) indicates the relative strength for each species-season combination. For example, both bighorn sheep and mountain goats were hypothesized to have a negative relationship with elevation in winter, but the association was expected to be greater for bighorn sheep than for mountain goats, as indicated by boldface type for bighorn sheep. Li, linear; Sq, quadratic; Ps, natural log/pseudothreshold.

$\dagger$ Circular buffer in meters.

\$The 30-m SlopeVar was generated using a 30-m neighborhood analysis of a 10-m resolution slope raster and served as the base layer to generate SlopeVar at the larger spatial grains. 
combination. With a final list of covariates that balanced the univariate results with collinearity, we fit a single multivariate model for each individual and generated a single population-averaged model for each species-season group to estimate inverse variance weighted mean coefficients $(\beta \mathrm{s}$; Murtaugh 2007, DeCesare et al. 2012) across individuals $i$ for each species $j$, season $s$, and covariate $k$ :

$$
\hat{\beta}_{j s k}=\sum_{i=1}^{N} w_{i j s k} \hat{\beta}_{i j s k}
$$

where $w_{i j s k}$ 's represented the seasonal individual parameter weights estimated as

$$
w_{i j s k}=\frac{1 /\left[\operatorname{SE}\left(\hat{\beta}_{i j s k}\right)\right]^{2}}{\sum_{i=1}^{N}\left(1 /\left[\operatorname{SE}\left(\hat{\beta}_{i j s k}\right)\right]^{2}\right)}
$$

and standard errors are estimated as

$$
\mathrm{SE}\left(\hat{\beta}_{j s k}\right)=\sqrt{\frac{\sum_{i=1}^{N} w_{i j k k}\left(\hat{\beta}_{i j k}-\hat{\beta}_{j k s}\right)^{2}}{N-1}} .
$$

Second, we characterized the seasonal realized niches in geographic space by extrapolating the population-averaged RSF within the sympatric study area. We estimated the relative probability of use with the exponential RSF,

$$
\widehat{w}(x)=\exp \left(\hat{\beta}_{1} x_{1}+\hat{\beta}_{2} x_{2}+\ldots+\hat{\beta}_{n} x_{n}\right)
$$

where $\beta$ s are the coefficients of the effects of the covariates, $X_{i}$, on $\widehat{w}(x)$, the relative probability of use. The predicted RSF values were then rescaled between 0 and 1 with a linear stretch (Johnson et al. 2004, DeCesare et al. 2012). We evaluated the predicted values by calculating a correlation coefficient between the number of used GPS locations within 10 equalarea RSF bins, which characterized the available distribution of predicted values from 500,000 random locations within the study area (Boyce et al. 2002, DeCesare et al. 2012).

Last, we characterized the distributions of the minimum resolution and linear form of each niche component from the used GPS locations using violin plots (Wickham 2016). This provided an assessment of the seasonal niches solely reliant on the used locations without incorporating multiple spatial grains, functional forms, or an "available" sample. All spatial and statistical analyses were conducted in R ( R Core Team 2016).

\section{Evaluating niche similarity}

We evaluated seasonal niche similarity of bighorn sheep and mountain goat niches characterized with RSFs in niche and geographic space as well as the characterizations with used locations. Within niche space, we evaluated the confidence interval overlap of the averaged model coefficients for each species as well as the relative similarity according to predictive plots over the range of each covariate. Following recent advances in ecological niche modeling (Warren et al. 2008, Broennimann et al. 2012), we evaluated niche similarity in geographic space by comparing model predicted values from each species with Schoener's $D$ metric (Schoener 1968),

$$
D=1-\frac{1}{2}\left(\sum_{i j}\left|z_{1 i j}-z_{2 i j}\right|\right),
$$

where $z_{1}$ and $z_{2}$ represented the stretched population-level RSFs rescaled so that the sum of the predicted values was the same for both species (Broennimann et al. 2012) and $i$ and $j$ indexed rows and columns, respectively. The $D$ metric ranges between 0 (dissimilar niches) and 1 (identical niches) and can be used with any niche model that produces suitability measures in geographic space (Warren et al. 2008). Moreover, Schoener's $D$ provides a relatively simple measure with a long history of use in both dietary and space use studies and is typically applied to measures of relative use (Schoener 1968, Warren et al. 2008), such as the relative RSF estimated from a used-available design (Manly et al. 2002).

In addition, we evaluated the ability of one species' niche to predict the other species in geographic space by comparing single bin values of one species to the corresponding grid cells of the other species. More specifically, for each binned RSF value of bighorn sheep, we calculated the proportion of mountain goat bin values that corresponded with a single bighorn sheep bin in geographic space. The method is insensitive to the species direction (e.g., bighorn sheep to mountain goats or mountain goats to bighorn sheep), as one is a transformation of the other. In the case of niche equivalency (e.g., $D=1$ ), there would be perfect alignment between the realized niches in geographic space (Fig. 2). In contrast, dissimilar niches would appear random with an equivalency similar to that expected by chance (Fig. 2).

Last, for the niche characterization with used locations, we compared the distribution shape and mean for each niche component to identify relative differences among species.

\section{RESUlts}

\section{Data collection and censoring}

Capture efforts began in the spring of 2012 and continued to the winter of 2016, resulting in the instrumentation of 34 female bighorn sheep (BHS) and 23 mountain goats (MTG; 15 females, 8 males). We censored a single individual of each species monitored $\leq 32 \mathrm{~d}$ and an additional 790 locations with a HDOP $>10$ and 5,318 locations with a horizontal error estimate $>60 \mathrm{~m}$. Our final seasonal samples contained $31,229$ (BHS $=18,997$, MTG $=12,232)$ summer locations from 51 individuals $(\mathrm{BHS}=29, \mathrm{MTG}=22$ ) and 65,237 $(\mathrm{BHS}=43,548, \mathrm{MTG}=21,689)$ winter locations from 55 individuals $(\mathrm{BHS}=33, \mathrm{MTG}=22 ;$ Appendix $\mathrm{S} 4)$. The availability extent $\left(4,389 \mathrm{~km}^{2}\right)$ encompassed all GPS locations and was buffered by $962 \mathrm{~m}$, representing the 95 th percent quantile of sequential step lengths (Fig. 1).

\section{Seasonal niche characterization and similarity}

Niche space.-For most covariates within the univariate models there were appreciable differences between grains and forms that varied within and among species and season. 
A

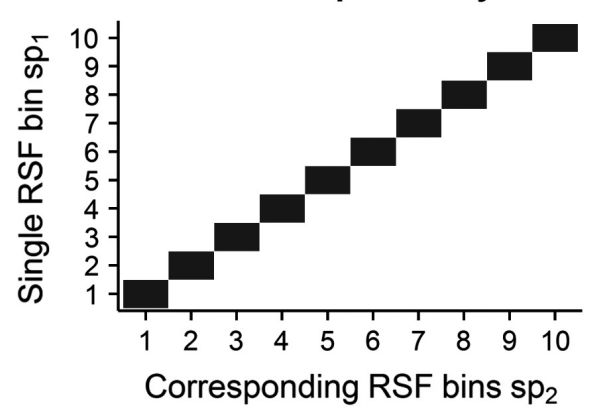

B

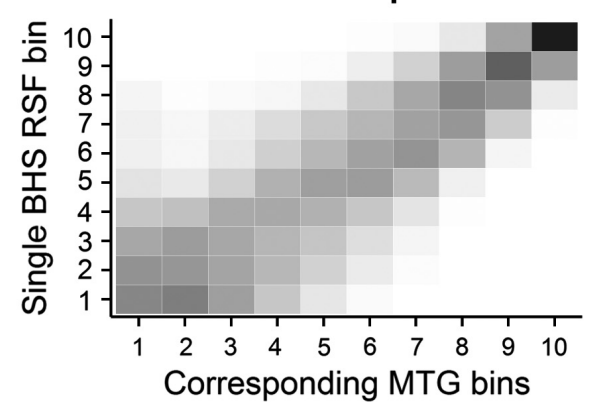

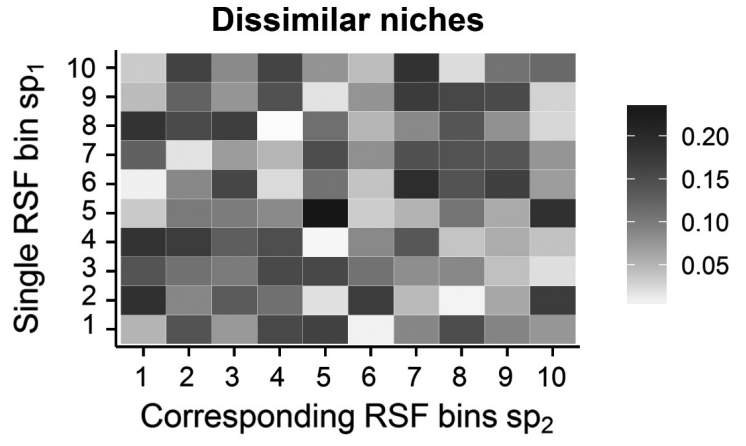

Observed overlap: Winter

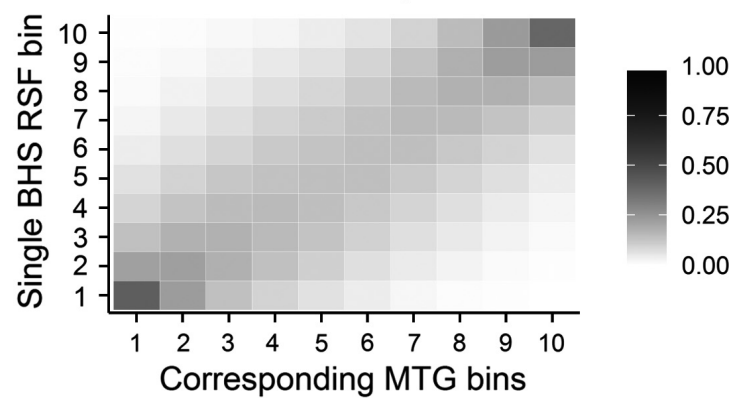

FIG. 2. (A) Schematic depiction of the two extremes when comparing niche overlap. Each cell represents the proportion of $\operatorname{species}_{2}\left(\mathrm{sp}_{2}\right)$ resource selection function (RSF) bins within a single bin of species ${ }_{1}\left(\mathrm{sp}_{1}\right)$. When two niches are equivalent (e.g., Schoener's $\left.D=1\right)$, there will be perfect alignment between the RSF bins. In contrast, dissimilar niches (e.g., $D=0$ ) will appear random with an overlap similar to that expected by chance. Note the different coloring scales between the two panels. (B) Cell by cell comparison of the observed seasonal niche overlap of sympatric bighorn sheep and mountain goats in the northeast GYA. Each cell represents the proportion of RSF values from one species that corresponds to a single RSF bin of the other species.

While the majority of covariates had a clearly top-ranked form and/or grain within the species-season groupings, some grains and/or forms were only marginally higher ranked (Appendix S5). When moving from univariate to multivariate models, we changed the summer DST definition from $40^{\circ}$ to $50^{\circ}$ for bighorn sheep, and changed the grain of SlopeVar from 1,000 to $30 \mathrm{~m}$ for mountain goats to reduce collinearity with slope (Table 2; Appendix S5). In winter, we removed SWE, which was collinear with elevation for both species (Table 2; Appendix S5).

There was strong similarity between bighorn sheep and mountain goat weighted mean coefficients in both seasons (Fig. 3) and similar relationships with each covariate (Appendix S6). The summer niches of both species were characterized by relatively cool aspects with little canopy cover on steep and rugged slopes at high elevations (Fig. 3). Slope was the most influential covariate for both species and showed the strongest evidence of niche partitioning. In contrast to our hypotheses, the standardized coefficients suggested the summer bighorn sheep niche was characterized by steeper slopes than that of mountain goats (Fig. 3; Appendix S6). Moreover, both the linear and quadratic coefficients of slope were positive, indicating positive and accelerating selection for steep slopes by both species rather than an optimal mid-level value for slope. The winter niches of both species were characterized by warmer, southwest slopes, at relatively low elevations with minimal canopy cover, and steep and rugged slopes (Fig. 3; Appendix S6). Slope was the most influential covariate in winter, yet in contrast to summer, indicated the mountain goat niche was
TABLE 2. The top-ranked covariate grains and forms used to define the realized Grinnellian niches of sympatric bighorn sheep and mountain goats, northeast GYA, 2012-2017.

\begin{tabular}{lrrrrr}
\hline \hline & \multicolumn{2}{c}{ BHS } & & \multicolumn{2}{c}{ MTG } \\
\cline { 2 - 3 } \cline { 5 - 6 } Covariate & Grain & Form & & Grain & Form \\
\hline Summer & & & & \\
Aspect & 30 & $\mathrm{Li}$ & 30 & $\mathrm{Li}$ \\
CanCov & 500 & $\mathrm{Li}$ & 1,000 & $\mathrm{Li}$ \\
DST50 & 30 & $\mathrm{Li}$ & 30 & $\mathrm{Li}$ \\
Ele & 30 & $\mathrm{Li}$ & 30 & $\mathrm{Li}$ \\
NDVI & 250 & $\mathrm{Li}$ & 1,000 & $\mathrm{Li}$ \\
Slope & 1,000 & $\mathrm{Sq}$ & 1,000 & $\mathrm{Sq}$ \\
SlopeVar & 30 & $\mathrm{Li}$ & 30 & $\mathrm{Ps}$ \\
Winter & 30 & $\mathrm{Li}$ & 30 & $\mathrm{Li}$ \\
Aspect & 30 & $\mathrm{Li}$ & 500 & $\mathrm{Li}$ \\
CanCov & 30 & $\mathrm{Li}$ & 30 & $\mathrm{Li}$ \\
DST50 & 30 & $\mathrm{Li}$ & 30 & $\mathrm{Li}$ \\
Ele & 1,000 & $\mathrm{Li}$ & 1,000 & $\mathrm{Li}$ \\
NDVI & 1,000 & $\mathrm{Sq}$ & 30 & $\mathrm{Sq}$ \\
Slope & 30 & $\mathrm{Ps}$ & 30 & $\mathrm{Ps}$ \\
SlopeVar & & & & \\
\hline
\end{tabular}

characterized by steeper slopes than that of bighorn sheep. Bighorn sheep also tended to use relatively low elevations compared to mountain goats and had a stronger avoidance of canopy cover in both seasons (Fig. 3).

Geographic space.-Spearman rank correlations between the RSF bins and the frequency of used locations were high 


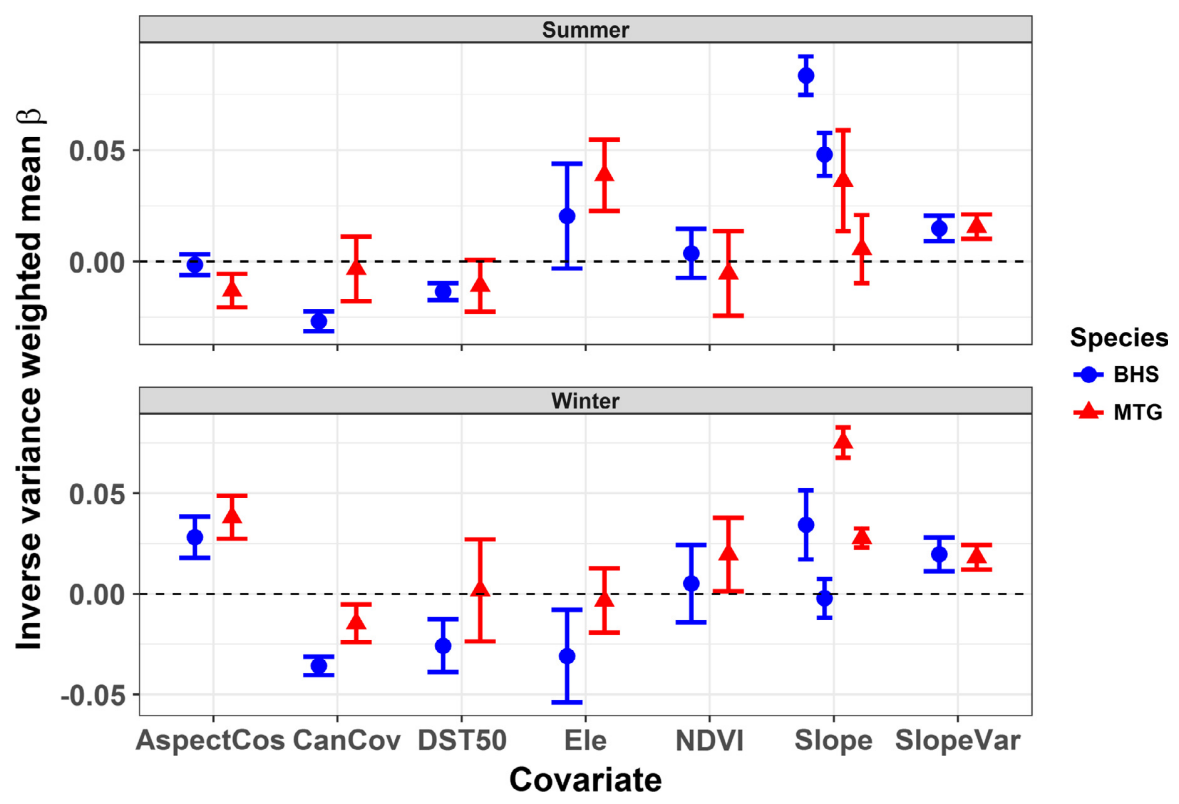

FIG. 3. Inverse variance weighted mean coefficients (and 95\% CIs) estimated from individual resource selection models using a twostage framework (with scaled covariates) for sympatric bighorn sheep and mountain goats in the northeast GYA. Both the linear and quadratic terms are shown respectively for the slope covariate Abbreviations are described in Table 1.

(mean $r_{\mathrm{s}} \geq 0.87$, mean $P \leq 0.02 ;$ Appendix S7), indicating strong predictive performance. Not surprisingly, our extrapolations from niche to geographic space indicated similar niches between native bighorn sheep and introduced mountain goats (summer $D=0.88$, winter $D=0.87$; Fig. 4 ). The summer niches of both species were centered on rugged and steep terrain at high elevations that was contiguously distributed within the study area (Fig 4). The strong similarity between summer niches was also evident in the cellby-cell comparisons of the RSF bins, especially at high bin values (Fig. 2). In winter, both species trended to lower elevations, although the stronger effect of elevation for bighorn sheep was evident in the geographic niche representations. Relative to mountain goats, the winter bighorn sheep niche encompassed more low-elevation valley bottoms, although the two niches did overlap on steep, mid-elevation slopes characteristically used by mountain goats. While the bighorn sheep niche was relatively contiguous at low elevations, the mountain goat niche was patchily distributed throughout the study area and largely excluded valley bottoms (Fig. 4). The discrepancies at low elevations also contributed to the larger range of RSF bins of one species associated with each bin of the other species in winter (Fig. 2).

Used locations. - Our characterizations of seasonal niches from used GPS locations indicated similar distributions for the niche components (Fig. 5). Differences were most pronounced for snow water equivalent, which was notably higher for mountain goats relative to bighorn sheep, although this covariate was removed from model predictions because of collinearity with elevation. The remaining niche components showed similar seasonal patterns, with slight differences in the distribution means among species (Fig. 5).

\section{Discussion}

Our work applied the niche partitioning hypothesis to sympatric mountain ungulates, native bighorn sheep and introduced mountain goats, and indicated similar niches in both niche and geographic space. While both species occur on sympatric native ranges throughout portions of western North America, this study was the first empirical analysis of their sympatric realized niches and, importantly, involved native and introduced populations where there is an immediate need to better understand the ecological consequences of introduced mountain goats. Using a variety of methods across niche and geographic space, our work indicated limited evidence of seasonal niche partitioning. Slope was the dominant niche component in both seasons and indicated bighorn sheep occurred on steeper slopes than mountain goats in summer while mountain goats occurred on steeper slopes in winter. In addition, canopy cover was more strongly avoided by bighorn sheep in both seasons and bighorn sheep tended to occur at lower elevations than mountain goats in winter. The remaining niche components were similar among species, resulting in niches that were highly correlated in geographic space.

The conceptual framework initially put forth by Adams et al. (1982) hypothesized mountain goats would occupy the extreme end of habitat-related niche components with partial overlap on sympatric ranges, but did not specify potential seasonal differences. We hypothesized that the Adams et al. (1982) conceptual model would have more relevance in winter and reflect differing wintering strategies between the two species. Regionally, mountain goats tend to select steep slopes at mid-elevations that more readily shed snow (Chadwick 2002, Lowrey et al. 2017), while bighorn sheep move to lower elevations with less snow accumulation (FestaBianchet 1988). While we did see mountain goats using 

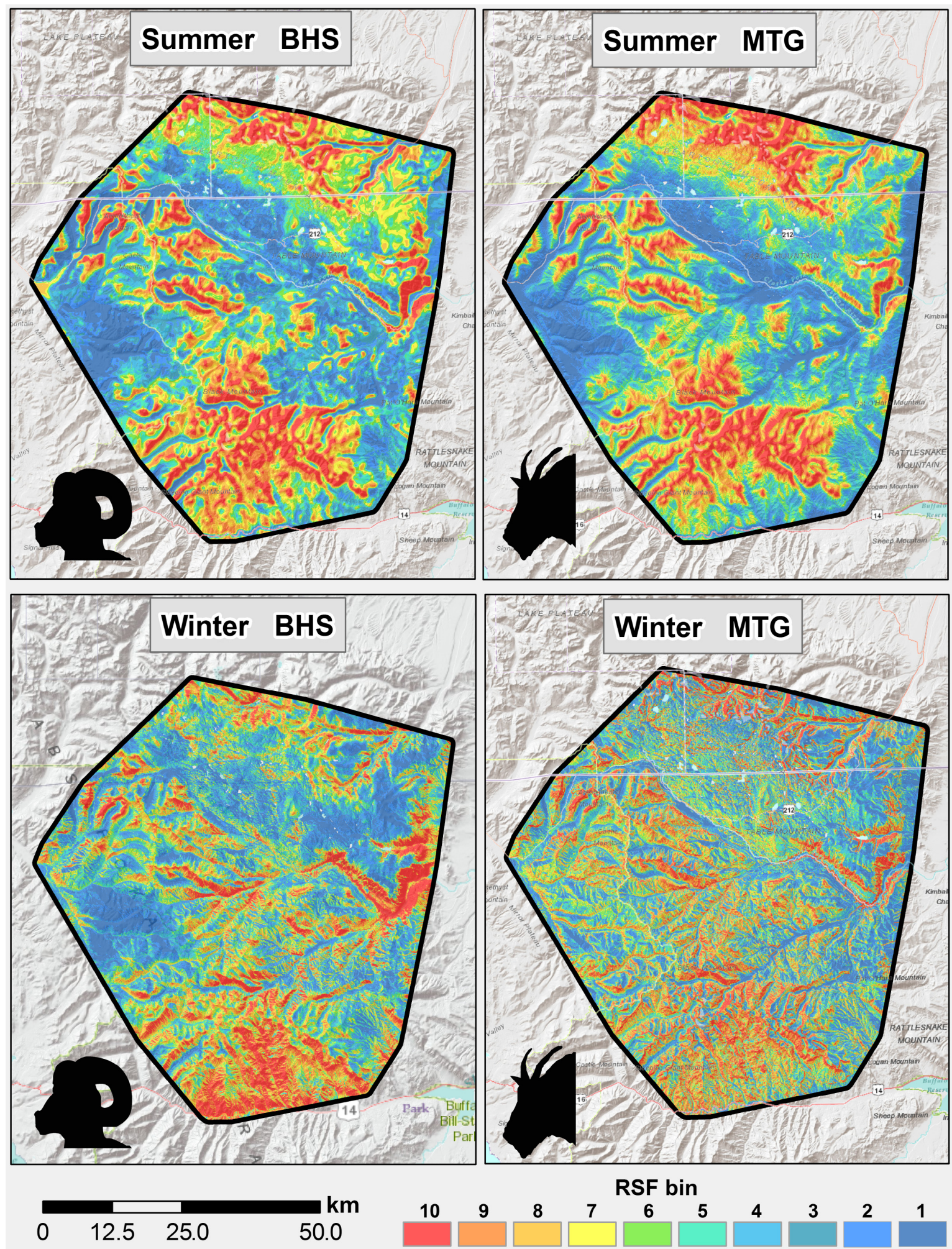

RSF bin

FIG. 4. Seasonal geographic realized niche predictions for sympatric bighorn sheep and mountain goats in the northeast, GYA.

relatively steeper slopes in winter, the pronounced differences in slope did not result in notable partitioning in geographic space when combined with the remaining niche components, a finding that was contrary to our hypotheses. In contrast, the niche similarities in summer aligned with our hypotheses as both species tended to use rugged, mountain environments at high elevations. Given the strong seasonal overlap described in our results, we are unable to rule out the possibility of direct or indirect competition. Nonetheless, there are a number of factors that may help to 

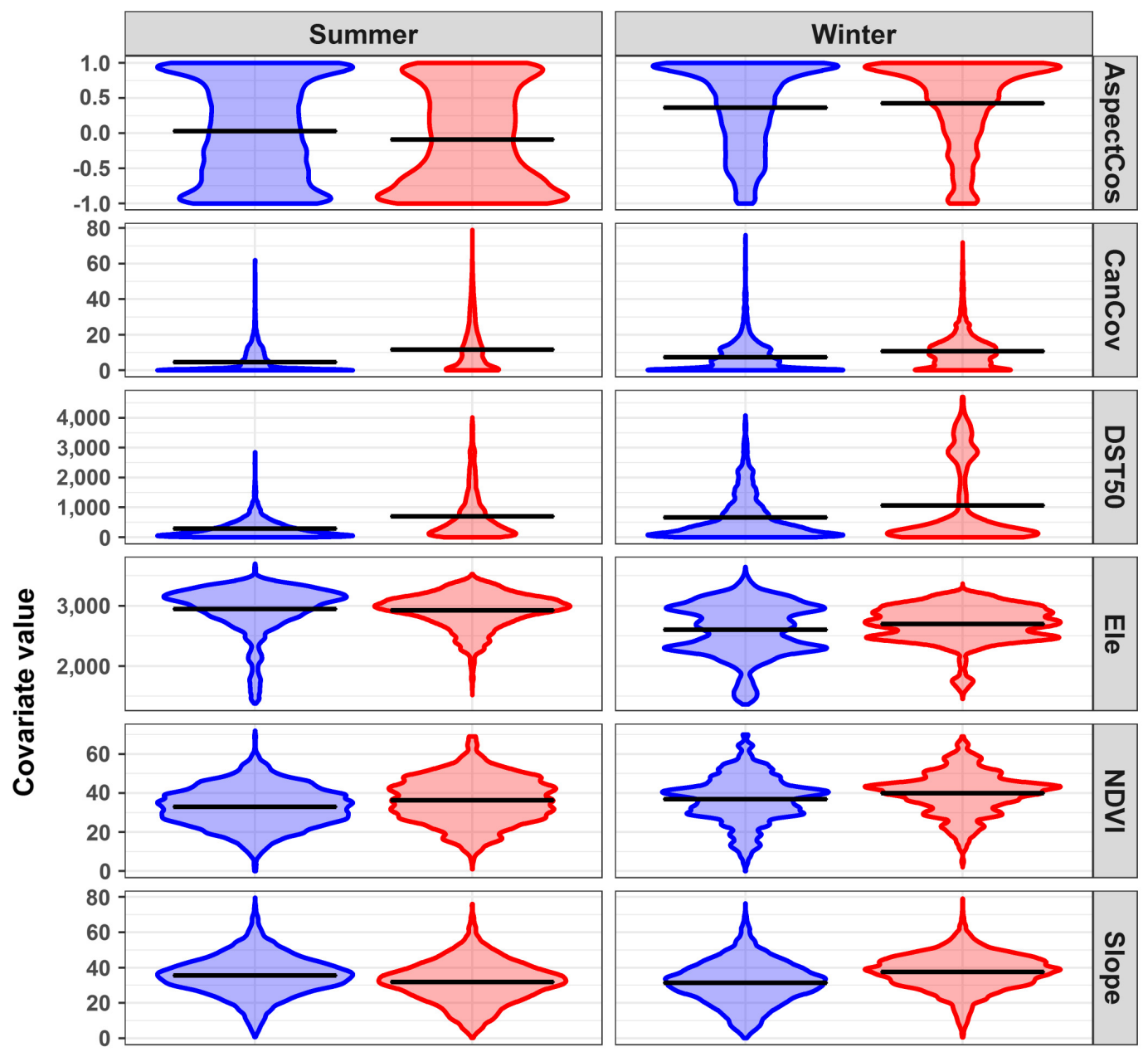

\section{Species $\square$ BHS MTG}
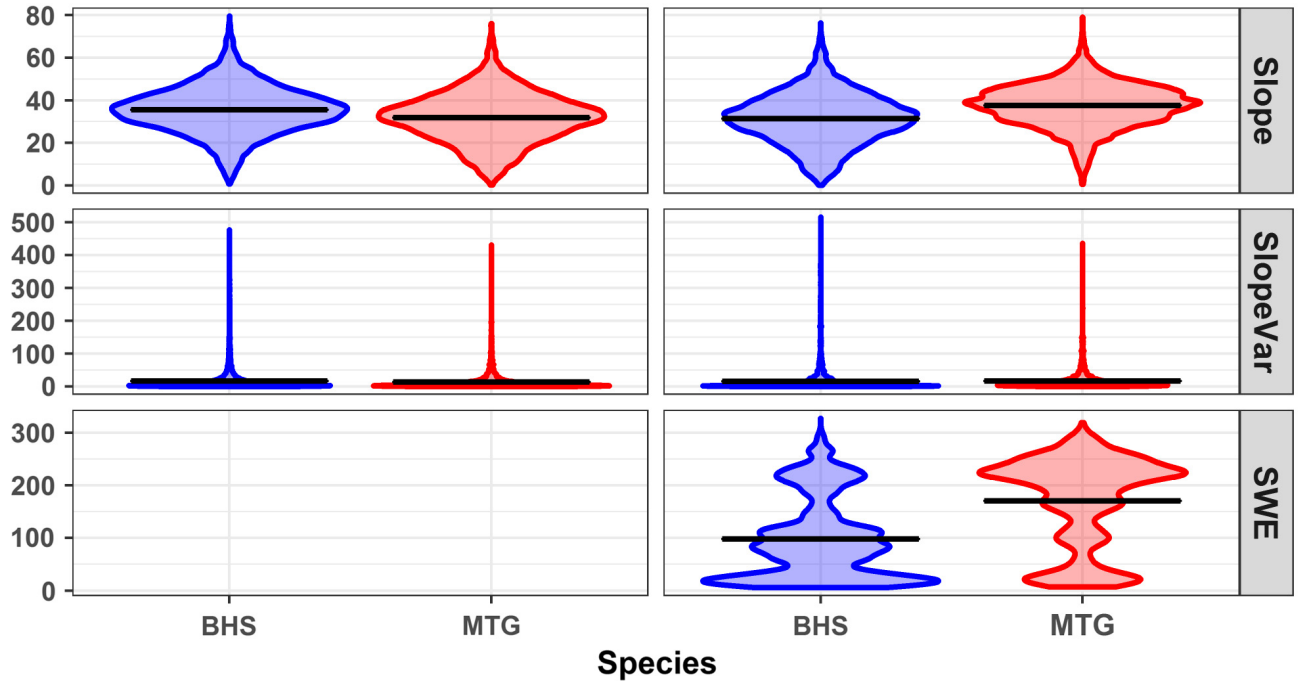

FIG. 5. Violin plots showing the distribution of niche components associated with used GPS locations from sympatric bighorn sheep and mountain goats in the northeast, GYA. Distribution means are shown with solid black lines. Abbreviations are described in Table 1.

explain the lack of niche partitioning among sympatric mountain ungulates in our study area.

Space use is regarded as an important axis along which a broad range of taxa partition resources (Schoener 1974, Stewart et al. 2002, Jenkins et al. 2007). Nonetheless, species that overlap along the spatial niche axis may yet reduce competition by partitioning niches along temporal or dietary axes (Pianka 1974, Schoener 1974). Both bighorn sheep and mountain goats have evolved as habitat specialists. Given the relatively narrow habitat type within which both species occur, the dietary or temporal niche axes may provide more flexibility in niche partitioning. We are not aware of any studies that have investigated the temporal patterns of sympatric mountain ungulates. However, previous studies have added some support to the presence of dietary partitioning, but definitive conclusions remain elusive. In a review paper, Laundré (1990) concluded that both species have similar diets when broad vegetation classes (grasses, forbs, browse) were compared across studies of allopatric populations, but that diet overlap was reduced when the two species were sympatric. Importantly, however, Laundré (1990) also emphasized the need for additional studies and the inability to draw 
rigorous conclusions with the available data. Working within a northern subset of our study area, Varley (1994) documented dissimilar summer feeding areas between bighorn sheep and mountain goats, but did not examine their niches as defined here and was again limited to broad vegetation classes within a narrow temporal window.

Although working within a different "niche" framework, the Varley (1994) results highlight scale as an important lens through which to interpret our results. We were specifically interested in population niches and maintaining the ability to directly compare seasonal niches among species. As a result, we defined availability equally for all individuals and seasons to negate the influence of the available sample on conclusions regarding seasonal niche characteristics and niche partitioning (Beyer et al. 2010). Moreover, the population-level inferences are often most relevant to regional managers tasked with monitoring mountain goat expansion and mitigating for any potential negative impacts posed to native species, including bighorn sheep. Similar analyses of sympatric and allopatric species conducted across multiple scales have shown differential patterns in resource selection and niche overlap (Jenkins et al. 2007, DeCesare et al. 2012). While our population-level study design indicated similar seasonal niches, a finer-scale analysis (e.g., within home ranges) in conjunction with behavioral data may indicate niche partitioning at a scale not captured with our broad sample of availability, for example, the dissimilar summer feeding areas described by Varley (1994). Nonetheless, we feel the descriptive niche characterizations from the used GPS locations further support our findings of niche similarity, irrespective of scale and availability (Fig. 5).

While our results indicated similar niches, equating niche similarity and overlap with competition can be "dubious and misleading" (Pianka 1974). By definition, resources need to be limiting for niche overlap to result in competition (Gause 1934). Given present densities and distributions of both species in the northeast GYA, the observed niche overlap may not result in exploitive competition, especially in summer when there is an abundance of forage within mountain environments. Niche overlap among sympatric species has been documented in other mountain ungulates, including Asiatic ibex (Capra ibex) and blue sheep (Pseudois nayaur) in northern India (Namgail 2006), as well as chamois (Rupicapra rupicapra) and mouflon (Ovis gmelini musimon) in the northern French Alps (Darmon et al. 2012). In both studies, researchers found positive associations between sympatric species using abiotic niche components (e.g., terrain features) to describe and predict overlap in niche (Namgail 2006) and geographic space (Darmon et al. 2012). While the generalities are not entirely consistent among published studies of mountain ungulates (Namgail et al. 2004), the relatively low densities at which mountain ungulates exist on the landscape may minimize the negative effects of overlapping niches, therefore negating the need to partition resources in response to competition.

While our data were broadly overlapping within the study area, because of the difficulty in capturing both species we did not have a large sample of animals that overlapped in both space and time, and were unable to evaluate the direct displacement of one species by the other (i.e., interference competition). Nonetheless, mountain goats have been shown to exhibit a relatively high degree of intraspecific aggressiveness (Côté 2000, Chadwick 2002), and were observed usurping space and resources from bighorn sheep in 39 of $107(36.5 \%)$ interactions in a Colorado study (Reed 2001). While mountain goats appear to dominate in interspecific interactions, it is unclear how often these events occur within our study area. Direct encounters were prohibitively difficult to observe within our expansive study area, especially in winter when bighorn sheep and mountain goats were more likely to encounter one another because of the general reduction of suitable habitat. While data on the interspecific interactions of mountain ungulates remains limited, remote trigger camera traps placed at known mineral licks may help to further our understanding of the behavioral interactions between bighorn sheep and mountain goats when seeking a shared, but limited, resource.

Interspecific interactions between bighorn sheep and mountain goats may also be mediated by seasonal movement strategies. Although all niche extrapolations showed strong predictive performance when averaged across individuals, our validation plots highlighted four bighorn sheep for which the winter model had relatively poor predictive performance (Appendix S7). All four sheep remained at relatively high elevations during winter months and may be indicative of varying wintering strategies within regional bighorn sheep. While continued research is beginning to describe bighorn sheep seasonal movements, management surveys have previously located bighorn sheep wintering at high elevations (D. E. McWhirter, unpublished data). Moreover, in the southwest GYA, Courtemanch et al. (2017) recently described an "abbreviated migration" in which bighorn sheep within Grand Teton National Park remain on high-elevation, windswept ridgelines for most of the year, but descend $500 \mathrm{~m}$ in spring to gain access to newly emergent forage approximately $30 \mathrm{~d}$ prior to spring green up on the high-elevation winter and summer ranges. Seasonal movements and wintering strategies may be an important component of mountain ungulate niche partitioning. While bighorn sheep that undergo elevational migrations between low-elevation winter ranges and high-elevation summer ranges may naturally separate from mountain goats, bighorn sheep that remain at high elevations year-round may increase spatial overlap with nonnative mountain goats during the winter months.

At present, the observed spatial and niche overlap does not appear to be negatively impacting demographics as both species have maintained positive growth rates over the period of mountain goat expansion (Flesch et al. 2016, Butler et al. 2017). However, given the similarity in seasonal niches, it is likely that mountain goats will continue to increase their spatial overlap with native bighorn sheep and increase densities where the two species are sympatric presently. While the relative abundance of forage and contagious habitat in summer is unlikely to result in the limited resources required for competition, expanding mountain goats may still negatively impact bighorn sheep in winter when resources are less available and patchily distributed. Moreover, similar niche requirements in winter may increase the likelihood of interspecific interactions, which in addition to displacing bighorn sheep, may also transmit novel respiratory pathogens between species (Gross 2001) and further hinder bighorn sheep restoration efforts. In contrast to our winter hypotheses and previous conceptual 
models, our results did not indicate meaningful niche partitioning. Our results suggest that reducing densities of mountain goats in hunted populations that are sympatric with bighorn sheep and impeding their expansion may reduce the possibility of competition and disease transfer. Given the current uncertainties regarding the possibility of partitioning at finer spatial scales and along dietary or temporal niche axes, the similarities of the population niches presented here suggests a conservative management approach that liberalizes harvest of introduced mountain goats outside of Yellowstone National Park, where hunting was banned in 1894, may be warranted. In concert, additional studies that specifically investigate partitioning at finer scales and along other niche axes will help to further evaluate the possibility of competition and can be used to inform an adaptive management approach. Where applicable and/or feasible, experimental removal of mountain goats would further our understanding of niche flexibility across a broad range with both sympatric and allopatric conditions, and inform interspecific competition between sympatric populations.

As the availability and accessibility of spatial data continues to revolutionize ecological research, we see an opportunity to build from single-species habitat maps to applied interspecific research with broad ecological relevance. Our work presented herein exemplifies one such possibility and integrates applicable tools from the habitat suitability and niche modeling fields to evaluate seasonal niche partitioning of native bighorn sheep and introduced mountain goats in the GYA.

\section{ACKNOWLEDGMENTS}

Primary funding for this work was provided by the National Park Service (Yellowstone and Grand Teton National Parks), Wyoming Game and Fish Department, Canon USA Inc. (via the Yellowstone Park Foundation), Greater Yellowstone Coordinating Committee, Shoshone National Forest, and Wyoming Governor's Big Game License Coalition. Additional funds and scholarships were provided by Montana State University, Wyoming Wild Sheep Foundation, Montana Wild Sheep Foundation, Wild Sheep Foundation, International Order of Rocky Mountain Goats, and the Kevin Hurley Wild Sheep Biology Award. J. T. Paterson provided insightful discussion and technical support. We thank the Wyoming Game and Fish Department for vital logistical and field support, including the successful capture of study animals and collar recovery. We thank L. McNew, J. Rotella, L. Adams, and two anonymous reviewers for thoughtful comments on earlier drafts of the manuscript.

\section{Literature Cited}

Adams, L. G., K. L. Risenhoover, and J. A. Bailey. 1982. Ecological relationships of mountain goats and Rocky Mountain bighorn sheep. Northern Wild Sheep and Goat Council 3:9-22.

Beyer, H. L., D. T. Haydon, J. M. Morales, J. L. Frair, M. Hebblewhite, M. Mitchell, and J. Matthiopoulos. 2010. The interpretation of habitat preference metrics under use-availability designs. Philosophical Transactions of the Royal Society B 365:2245-2254.

Boyce, M. S., P. R. Vernier, S. E. Nielsen, and F. K. A. Schmiegelow. 2002. Evaluating resource selection functions. Ecological Modelling 157:281-300.

Broennimann, O., et al. 2012. Measuring ecological niche overlap from occurrence and spatial environmental data. Global Ecology and Biogeography 21:481-497.

Buechner, H. K. 1960. The bighorn sheep in the United States, its past, present, and future. Wildlife Monographs 4:3-174.

Bunnefeld, N., L. Börger, B. van Moorter, C. M. Rolandsen, H. Dettki, E. J. Solberg, and G. Ericsson. 2011. A model-driven approach to quantify migration patterns: individual, regional and yearly differences. Journal of Animal Ecology 80:466-476.

Burnham, K. P., and D. R. Anderson. 2002. Model selection and multimodel inference: a practical information-theoretic approach. Springer, New York, New York, USA.

Butler, C. J., et al. 2017. Assessing respiratory pathogen communities in bighorn sheep populations: sampling realities, challenges, and improvements. PLoS ONE 12:e180689.

Cassirer, E. F., et al. 2017. Pneumonia in bighorn sheep: risk and resilience. Journal of Wildlife Management. https://doi.org/10. 1002/jwmg.21309

Chadwick, D. H. 2002. A beast the color of winter: the mountain goat observed. Bison Books, Lincoln, Nebraska, USA.

Chase, J. M., and M. A. Leibold. 2003. Ecological niches: linking classical and contemporary approaches. University of Chicago Press, Chicago, Illinois, USA.

Côté, S. D. 2000. Determining social rank in ungulates: a comparison of aggressive interactions recorded at a bait site and under natural conditions. Ethology 106:945-955.

Côté, S. D., and M. Festa-Bianchet. 2003. Mountain goat Oreamnos americanus. Pages 1061-1075 in G. A. Feldhamer, B. C. Thompson, and J. A. Chapman, editors. Wild mammals of North America: biology, management, and conservation. Johns Hopkins University Press, Baltimore, Maryland, USA.

Courtemanch, A. B., M. J. Kauffman, S. Kilpatrick, and S. R. Dewey. 2017. Alternative foraging strategies enable a mountain ungulate to persist after migration loss. Ecosphere 8:1-16.

Cushman, S. A., and D. O. Wallin. 2002. Separating the effects of environmental, spatial and disturbance factors on forest community structure in the Russian Far East. Forest Ecology and Management 168:201-215.

Darmon, G., C. Calenge, A. Loison, J.-M. Jullien, D. Maillard, and J.-F. Lopez. 2012. Spatial distribution and habitat selection in coexisting species of mountain ungulates. Ecography 35:44-53.

DeCesare, N. J., and D. H. Pletscher. 2006. Movements, connectivity, and resource selection of Rocky Mountain bighorn sheep. Journal of Mammalogy 87:531-538.

DeCesare, N. J., et al. 2012. Transcending scale dependence in identifying habitat with resource selection functions. Ecological Applications 22:1068-1083.

D'eon, R. G., and D. Delparte. 2005. Effects of radio-collar position and orientation on GPS radio-collar performance, and the implications of PDOP in data screening. Journal of Applied Ecology 42:383-388.

DeVoe, J. D., R. A. Garrott, J. J. Rotella, S. R. Challender, P. J. White, M. O'Reilly, and C. J. Butler. 2015. Summer range occupancy modeling of non-native mountain goats in the Greater Yellowstone Area. Ecosphere 6:1-20.

Elbroch, L. M., P. E. Lendrum, J. N. Newby, H. Quigley, and D. Craighead. 2013. Seasonal foraging ecology of non-migratory cougars in a system with migrating prey. PLoS ONE 8:e83375.

Elith, J., et al. 2006. Novel methods improve prediction of species' distributions from occurrence data. Ecography 29:129-151.

Festa-Bianchet, M. 1988. Seasonal range selection in bighorn sheep: conflicts between forage quality, forage quantity, and predator avoidance. Oecologia 75:580-586.

Fieberg, J., J. Matthiopoulos, M. Hebblewhite, M. S. Boyce, and J. L. Frair. 2010. Correlation and studies of habitat selection: Problem, red herring or opportunity? Philosophical Transactions of the Royal Society B 365:2233-2244.

Flesch, E. P., et al. 2016. Range expansion and population growth of non-native mountain goats in the Greater Yellowstone Area: challenges for management. Wildlife Society Bulletin 40:241-250.

Gause, G. F. 1934. The struggle for existence. Courier Corporation, North Chelmsford, Massachusetts, USA.

Grinnell, J. 1917. The niche-relationships of the California thrasher. Auk 34:427-433.

Gross, J. E. 2001. Evaluating effects of an expanding mountain goat population on native bighorn sheep: a simulation model of competition and disease. Biological Conservation 101:171-185. 
Hebblewhite, M., and D. T. Haydon. 2010. Distinguishing technology from biology: a critical review of the use of GPS telemetry data in ecology. Philosophical Transactions of the Royal Society B 365:2303-2312.

Hirzel, A. H., J. Hausser, D. Chessel, and N. Perrin. 2002. Ecological-niche factor analysis: How to compute habitat-suitability maps without absence data? Ecology 83:2027-2036.

Hirzel, A. H., and G. Le Lay. 2008. Habitat suitability modelling and niche theory. Journal of Applied Ecology 45:1372-1381.

Homer, C. G., J. A. Dewitz, L. Yang, S. Jin, P. Danielson, G. Xian, J. Coulston, N. D. Herold, J. D. Wickham, and K. Megown. 2015. Completion of the 2011 National Land Cover Database for the conterminous United States-representing a decade of land cover change information. Photogrammetric Engineering and Remote Sensing 81:345-354

Hutchinson, G. E. 1957. Concluding remarks. Cold Spring Harbor Symposia on Quantitative Biology 22:415-427.

Jenkins, D. A., J. A. Schaefer, R. Rosatte, T. Bellhouse, J. Hamr, and F. F. Mallory. 2007. Winter resource selection of reintroduced elk and sympatric white-tailed deer at multiple spatial scales. Journal of Mammalogy 88:614-624.

Johnson, C. J., D. R. Seip, and M. S. Boyce. 2004. A quantitative approach to conservation planning: using resource selection functions to map the distribution of mountain caribou at multiple spatial scales. Journal of Applied Ecology 41:238-251.

Keiter, R. B., and M. S. Boyce, editors. 1994. The Greater Yellowstone ecosystem: redefining America's wilderness heritage. Yale University Press, New Haven, Connecticut, USA.

Laforge, M. P., E. Vander Wal, R. K. Brook, E. M. Bayne, and P. D. McLoughlin. 2015. Process-focussed, multi-grain resource selection functions. Ecological Modelling 305:10-21.

Laundré, J. W. 1990. The status, distribution, and management of mountain goats in the Greater Yellowstone Ecosystem. NPS Order PX1200-8-0828. U.S. Department of the Interior, National Park Service, Yellowstone National Park, Mammoth, Wyoming, USA.

Lemke, T. O. 2004. Origin, expansion, and status of mountain goats in Yellowstone National Park. Wildlife Society Bulletin 32:532-541.

Lowrey, B., R. A. Garrott, H. M. Miyasaki, G. Fralick, and S. R. Dewey. 2017. Seasonal resource selection by introduced mountain goats in the southwest Greater Yellowstone Area. Ecosphere 8:1-20.

MacArthur, R. H. 1958. Population ecology of some warblers of northeastern coniferous forests. Ecology 39:599-619.

Manly, B. F., L. McDonald, D. Thomas, T. L. McDonald, and W. P. Erickson. 2002. Resource selection by animals: statistical design and analysis for field studies. Springer, New York City, New York, USA.

Marzluff, J. M., J. J. Millspaugh, P. Hurvitz, and M. S. Handcock. 2004. Relating resources to a probabilistic measure of space use: forest fragments and Steller's Jays. Ecology 85:1411-1427.

McWhirter, D. E. 2016. Cody region big game job completion report. Wyoming Game and Fish Department, Cheyenne, Wyoming, USA.

Montana Department of Fish, Wildlife and Parks [MFWP]. 2016. Bighorn sheep population estimates. fwp.mt.gov/fwpDoc.html? id $=74752$

Murtaugh, P. A. 2007. Simplicity and complexity in ecological data analysis. Ecology 88:56-62.

Namgail, T. 2006. Winter habitat partitioning between Asiatic ibex and Blue sheep in Ladakh, northern India. Journal of Mountain Ecology 8:7-13.

Namgail, T., J. L. Fox, and Y. V. Bhatnagar. 2004. Habitat segregation between sympatric Tibetan argali Ovis ammon hodgsoni and blue sheep Pseudois nayaur in the Indian Trans-Himalaya. Journal of Zoology 262:57-63.

NOHRSC. 2004. National operational hydrologic remote sensing center Snow Data Assimilation System (SNODAS) Data Products at NSIDC. National Snow and Ice Data Center, Boulder, Colorado, USA. https://doi.org/10.7265/n5tb14tc

Panzacchi, M., B. Van Moorter, O. Strand, L. E. Loe, and E. Reimers. 2014. Searching for the fundamental niche using individualbased habitat selection modelling across populations. Ecography 38:1-11.

Phillips, S. J., and M. Dudík. 2008. Modeling of species distributions with Maxent: new extensions and a comprehensive evaluation. Ecography 31:161-175.

Pianka, E. R. 1974. Niche overlap and diffuse competition. Proceedings of the National Academy of Sciences USA 71:2141-2145.

Pianka, E. R. 1981. Competition and niche theory. Pages 167-196 in R. M. May, editor. Theoretical ecology: principles and applications. Volume 8. Blackwell Scientific Publications, Oxford, UK.

R Core Team. 2016. R: a language and environment for statistical computing. R Foundation for Statistical Computing, Vienna, Austria. https://www.R-project.org/

Reed, D. F. 2001. A conceptual interference competition model for introduced mountain goats. Journal of Wildlife Management 65:125-128.

Sawyer, H., M. J. Kauffman, R. M. Nielson, and J. S. Horne. 2009. Identifying and prioritizing ungulate migration routes for landscape-level conservation. Ecological Applications 19:2016-2025.

Schoener, T. W. 1968. The Anolis lizards of Bimini: resource partitioning in a complex fauna. Ecology 49:704-726.

Schoener, T. W. 1974. Resource partitioning in ecological communities. Science 185:27-39.

Soberón, J., and M. Nakamura. 2009. Niches and distributional areas: concepts, methods, and assumptions. Proceedings of the National Academy of Sciences USA 106:19644-19650.

Spitz, D. B., M. Hebblewhite, and T. R. Stephenson. 2017. "MigrateR": extending model-driven methods for classifying and quantifying animal movement behavior. Ecography 40:788-799.

Stahler, D. R., D. W. Smith, and D. S. Guernsey. 2006. Foraging and feeding ecology of the gray wolf (Canis lupus): lessons from Yellowstone National Park, Wyoming, USA. Journal of Nutrition 136:1923S-1926S.

Stewart, K. M., R. T. Bowyer, J. G. Kie, N. J. Cimon, and B. K. Johnson. 2002. Temporospatial distributions of elk, mule deer, and cattle: resource partitioning and competitive displacement. Journal of Mammalogy 83:229-244.

USGS. 2009. United States Geological Survey National Elevation Dataset, Sioux Falls, South Dakota, USA. https://ta.cr.usgs.gov/ NED

USGS EROS Center. 2016. Remote sensing phenology. United States Geological Survey Earth Resources Observation and Science Center, Sioux Falls, South Dakota, USA. http://phenol ogy.cr.usgs.gov/

Varley, N. C. 1994. Summer-fall habitat use and fall diets of mountain goats and bighorn sheep in the Absaroka Range, Montana. Biennial Symposium of the Northern Wild Sheep and Goat Council 9:131-138.

Warren, D. L., R. E. Glor, M. Turelli, and D. Funk. 2008. Environmental niche equivalency versus conservatism: quantitative approaches to niche evolution. Evolution 62:2868-2883.

White, G. C., and R. A. Garrott. 1990. Analysis of wildlife radiotracking data. Academic Press, New York, New York, USA.

Wickham, H. 2016. ggplot2: elegant graphics for data analysis. Springer, New York, New York, USA 